\title{
The Design and Implementation of IC Card System Based on Digital Campus
}

\author{
Jiang $\mathrm{Li}^{1, \mathrm{a}}$ \\ ${ }^{1}$ Lijiang Teachers College, Lijiang, Yunnan Province, China \\ a249239551@qq.com
}

Keywords: Digital campus, Campus Cartoon, Design and Implementation.

\begin{abstract}
Campus id is an important part of the "digital campus" construction and foundation engineering, for the "digital campus" provides a comprehensive data collection network platform. This article from the campus id function module, provide a platform for other management system, fund settlement and system security four aspects discusses the campus all-in-one-card platform design.
\end{abstract}

\section{Introduction}

With the vigorously development of computer technology and network technology, campus card in school got in different stages of the popularization and promotion quickly. In the big tide of the digital campus construction, it has become one of the important parts of got the attention of the schools [1].

Digital campus one-card system USES the international advanced multilayer C/S and B/S hybrid architecture, application data exchange middleware technology development center, truly achieve the cartoon, library, netcom integrated IC card application system. Campus id system is build with the mode of $1+\mathrm{N}$, namely the "platform $+\mathrm{N}$ subsystems application" [1]. After building good campus id system platform, on the platform can be extended according to user's need to increase all kinds of application subsystems and don't need to change the platform, fully embodies the system with good scalability and compatibility.

\section{The Design of the CampusCard}

Campus in their daily work and life, regardless of teachers, or students often involves identification, permission validation, expense and other procedures, the advent of digital campus network construction and use of the above aspects of work more and more use of electronic information card [1]. For example, the library card library, cafeteria meal card, the school's students are, etc., can use electronic information card, but too many cards there are many drawbacks. We need an electronic card, but need a card can solve many problems, this is the "One Card" basic assumptions.

Standards-based network physical campus network platform, various application software systems, with a card can solve many fundamental problems in the daily management; improve management efficiency and management tools, to lay a solid foundation for the realization of information management technology [2]. According to a multi-purpose card, card use, real-time exchange, automated settlement principles, "one card in hand, around campus," the key middle schools will meet the needs of digital construction and purpose.

The overall objective of the card design is: the use of the physical platform of the campus network, and online data exchange with the update, the establishment of a safe, reliable, effective and practical, the combination of flexibility and performance ahead of "a multi-purpose card" application system [2], its topology shown in Figure 1. 


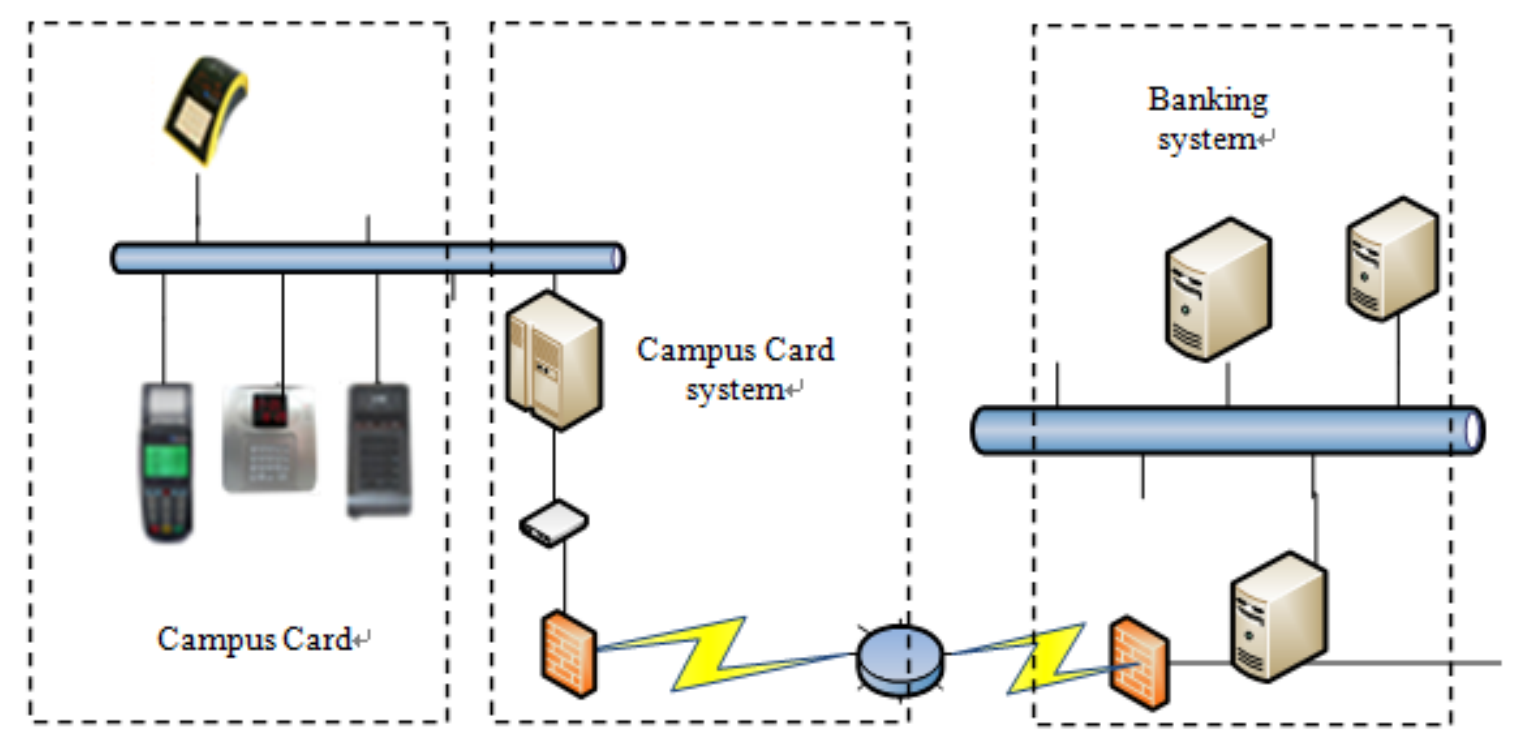

Fig.1 The campus one-card system network topology

Academy card system main platform. Campus card system established on the school campus network backbone platform, data center and regional business management divides into two parts, the data center core position in the school of information management, information network center), through the network are connected to the district management center [1]; Business management center placed in the open position of the districts (service center), to facilitate service for teachers and students.

Data center platform. Campus data center platform including database servers, application servers, disk arrays, etc. Servers typically consists of 2 servers a cluster and backup each other, USES the Shared disk array to provide data storage services. Campus id data center platform application software including financial data and status data center. Financial data center is used to store, manage, control, maintenance and backup of campus card financial information, including the cardholder account information, business information, transaction data, statistics, etc. [3], water table service way and later with invoicing, reconciliations and other financial services. Identity data center is used to store, manage, control, maintenance and backup system used the identity of the information needed, including one cartoon system of identity information, global dictionary information and photos, etc., and Taiwan after service provide identity information, in the form of updates and synchronization service [2]. Usually financial data center and identity data center is installed on the same server group.

Comprehensive business front platform. Comprehensive business front platform in the campus card system and IC card data center and the subsystem of the connection position, is the key to connect data center and subsystem hub, occupies an important position in the system, is the total control center of the campus one-card system [3].

Comprehensive business front platform is responsible for providing the global configuration parameter Settings and change, such as black and white list real-time synchronous real-time information management, the whole system security control of each subsystem, key generation and update management, to access campus card platform of various subsystems equipment condition monitoring, and other functions.

Circle transfer front platform. By bank transfer system were placed in the two front of the school and the bank machine, spread on campus transfer terminal (also called deposit machine) and the composition of communication networks. In the school end and bank end of a front machine, between the two front machines adopts special line or private network connection in the form of using TCP/IP protocol, through the application layer packet exchange realize transfers and other transactions [4]. Transfer frontend is bank transfer system in the key hub connecting schools and Banks, and a dedicated channel between Banks, through the school provide self-help transfer, transfer, settlement, reconciliation and other financial services. 
Integrated query front platform. Integrated query front platform is connected to the campus card center and all the query key hub between terminals, as a Web server, providing multimedia query functions, including online query, touch screen query, divided into personal enquiry centre for merchants and comprehensive center, information query, merchants can provide account information query [4], consumption record query, report the loss of STH, hang, modify, query password, public services, and other functions, query executes nobody duty $7 * 24$ hours service.

One cartoon system network structure. Campus all-in-one-card system belongs to the application of the application layer, network layer USES the TCP/IP protocol, support optical fiber, twisted-pair cable, wireless (433 m) and other kinds of cable, support $10 \mathrm{~m}$ and $100 \mathrm{~m}$ and $1000 \mathrm{~m}$ Ethernet technology, support routers, switches, hubs, firewalls, intrusion detection, encryption machine equipment such as various network devices [5].

The network environment of the campus one-card system can use two ways, one is based on campus network partition logical network segment, the VLAN technology to isolate one cartoon private network and campus network; Another kind is to set up private network, by applying the gateway with the campus network., in contrast, private network scheme improves security, disadvantage is high cost.

The card type. According to meet the needs of the development of IC card system in the future, the campus card with telecom with 13.56 MHZ non-contact rf card phone. Credit CARDS issued by Banks. Two card physical separation, a one-to-one relationship. From the function, the campus card is divided into registered card and not a registered card [5]. From the user, the campus card are divided into student card, staff card, card, temporary card from retiree, etc.

\section{The Application Realization of Campus Card}

Campus card front machine is connected to the bank and bank transfer key hub campus IC card system, also self-help transfer terminal server [5]. And self-help transfer terminal, together they implement, silver proofreading books, big bank transfer payment, bank card self-help binding, modify, query password, check balances, report the loss of function, shown in Fig.2.

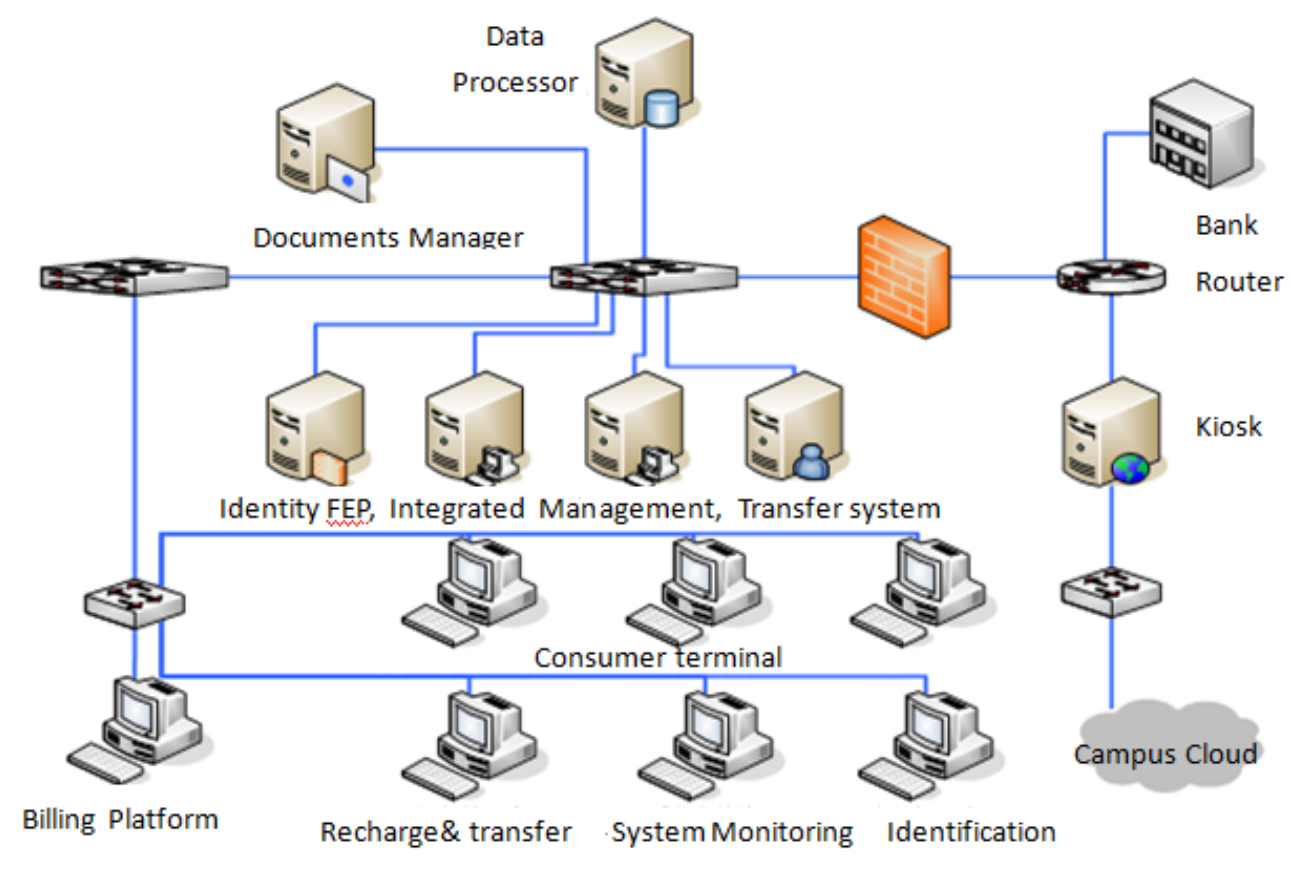

Fig.2 The system of campus card

Transfer function. Campus card can transfer money from bank cards, transfers and automatic transfers into a buffet. Self transfer initiated by the self-transfer terminal, launched trading after bank transfer to the bank dealt with separately FEP FEP data center and campus card, the results back to self-transfer terminal [6]. Automatic transferation initiated by the bank transfer front-end, get automatic transfer document and submits it to the bank front-end data center from campus card, and 
then analyze the results of the bank transfer FEP, made an application to the recorded data center campus card.

Reconciliation function. The comparison of the local money transfer transactions and bank transactions parties, if the parties appear inconsistent, the program automatically processed by the manual handling or negotiated [6].

Self-service payment function. Into the campus card and bank card kiosk self-service payment, the result resembles a campus card spending and bank transfer. Campus card self-service payment terminal transfers initiated by the self-initiated transaction card data center to the campus after campus FEP transfer process, the results back to self-transfer terminal [6]. Bank card self-service payment terminal transfers initiated by the self-initiated transactions to the bank after bank transfer FEP FEP processing, transfer the results back to the self-service terminal.

Campus card operation function. Supported by self-initiated transfer terminal campus card loss solution hanging, campus card password changes, modifications campus card spending limit, campus card balance inquiries, campus card transaction flow query, the results back to self-transfer terminal [6].

Bank card operation function. Support bank card transfer terminal initiated by the self-modifying code, bank card balance query, transfer the results back to the self-terminal.

System Configuration function. For self-service terminals, there are operator, bank connection, service information and other parameters of configuration options.

Query statistics function. On the transfer details, bank details, reconciliation details, details of the inquiry and the liquidation of the transfer information, the liquidation of statistical information. According to the query criteria input the selected information to search.

Monitor function. Monitor and control self-transfer terminal operation.

\section{Conclusion}

Campus Card is to use a card replace all the cards on campus, certificate, and ticket, to achieve a variety of different functions of intelligent management. The use of electronic money function cards, realize cashless settlement campus, campus regulate economic order. Lying covering school-wide private network card and the respective management information systems is a single entity, can use to solve the problem of information silos various management information systems, to create a good school environment, sharing of information resources, integration management. Design of campus card system must master plan, step by step, full use of existing resources. The system must have high security and stability, easy to manage and maintain. The system must also have good openness and scalability.

\section{References}

[1] G.H. Zhang, The university management information issues and countermeasures, and social development, 2005.pp.35-42.

[2] W.G. Jin and G.Q. Cheng, Security systems engineering design Xi'an: Xi'an University of Electronic Science and Technology Press, Jiaxing University, 2012, pp63-66.

[3] H.Y. Lin and Y.Chen, The intelligent community security system Beijing: Mechanical Industry Press, 2013,pp.43-47.

[4] P.Sun and L.G. Wang, The building intelligent security system, Beijing: Mechanical Industry Press, 2011, pp.45-47.

[5] J. Chen, Intelligent buildings weak equipment installation and commissioning, Higher Education Press, 2013.pp.22-26.

[6] A.Y. Wang, Smart card technology, Tsinghua University Press, 2015, pp.54-58. 